\title{
FAST ELECTRONS IN SMALL SOLAR FLARES
}

\author{
R. P. LIN \\ Space Sciences Laboratory, University of California, Berkeley, Calif. 94720, U.S.A.
}

\begin{abstract}
Because 5-100 keV electrons are frequently accelerated and emitted by the Sun in small flares, it is possible to define a detailed characteristic physical picture of these events. This review summarizes both the direct spacecraft observations of non-relativistic solar electrons, and observations of the X-ray and radio emission generated by these particles at the Sun and in the interplanetary medium. These observations bear on the basic astrophysical process of particle acceleration in tenuous plasmas. We find that in many small solar flares the $\sim 5-100 \mathrm{keV}$ electrons accelerated during flash phase constitute the bulk of the total flare energy. Thus the basic flare mechanism in these flares essentially converts the available flare energy into fast electrons. These electrons may produce the other flare electromagnetic emissions through their interactions with the solar atmosphere. In large proton flares these electrons may provide the energy to eject material from the Sun and to create a shock wave which could then accelerate nuclei and electrons to much higher energies.
\end{abstract}

\section{Introduction}

Two distinct acceleration processes are observed to occur in solar flares. The first is the flash phase acceleration of electrons to energies of $\sim 5$ to $\sim 100 \mathrm{keV}$. This flash phase acceleration occurs in many small solar flares. The second acceleration phase occurs only in a few large flares and accelerates protons and electrons to much higher energies. The bulk of this second phase acceleration occurs after the flash phase, and appears closely related to type II radio emission at the Sun and interplanetary shock waves observed at the orbit of the Earth. This review covers only the flash phase acceleration of electrons.

Non-relativistic electrons from the Sun were first directly observed near the beginning of the present solar cycle (Van Allen and Krimigis, 1965). Since then over 350 impulsive solar flare electron events have been observed, mostly from experiments on the IMP (Interplanetary Monitoring. Platform) series of spacecraft (Anderson and Lin, 1966; Lin and Anderson, 1967; Lin, 1970). Some examples are shown in Figure 1. These electrons are the energetic particle species most frequently emitted by the Sun, and often originate in small flares or sub-flares.

Non-thermal X-ray and radio emission (Figure 2) indicate the presence of energetic electrons at the Sun. Low frequency radio observations (Figure 3) from spacecraft have provided a way of tracing these electrons from the vicinity of the Sun to and beyond the orbit of the Earth (see Fainberg et al., 1972).

The $\sim 5-100 \mathrm{keV}$ electrons constitute the bulk of the total flare energy in many small solar flares. Thus to a first approximation those flares can be thought of as a mechanism to convert the available energy, presumably contained in the magnetic field, into energetic electrons. These electrons may also produce the optical flare emissions - EUV (Figure 2) and $\mathrm{H} \alpha$ emissions (Figure 4) - by their interactions with the solar atmosphere. 

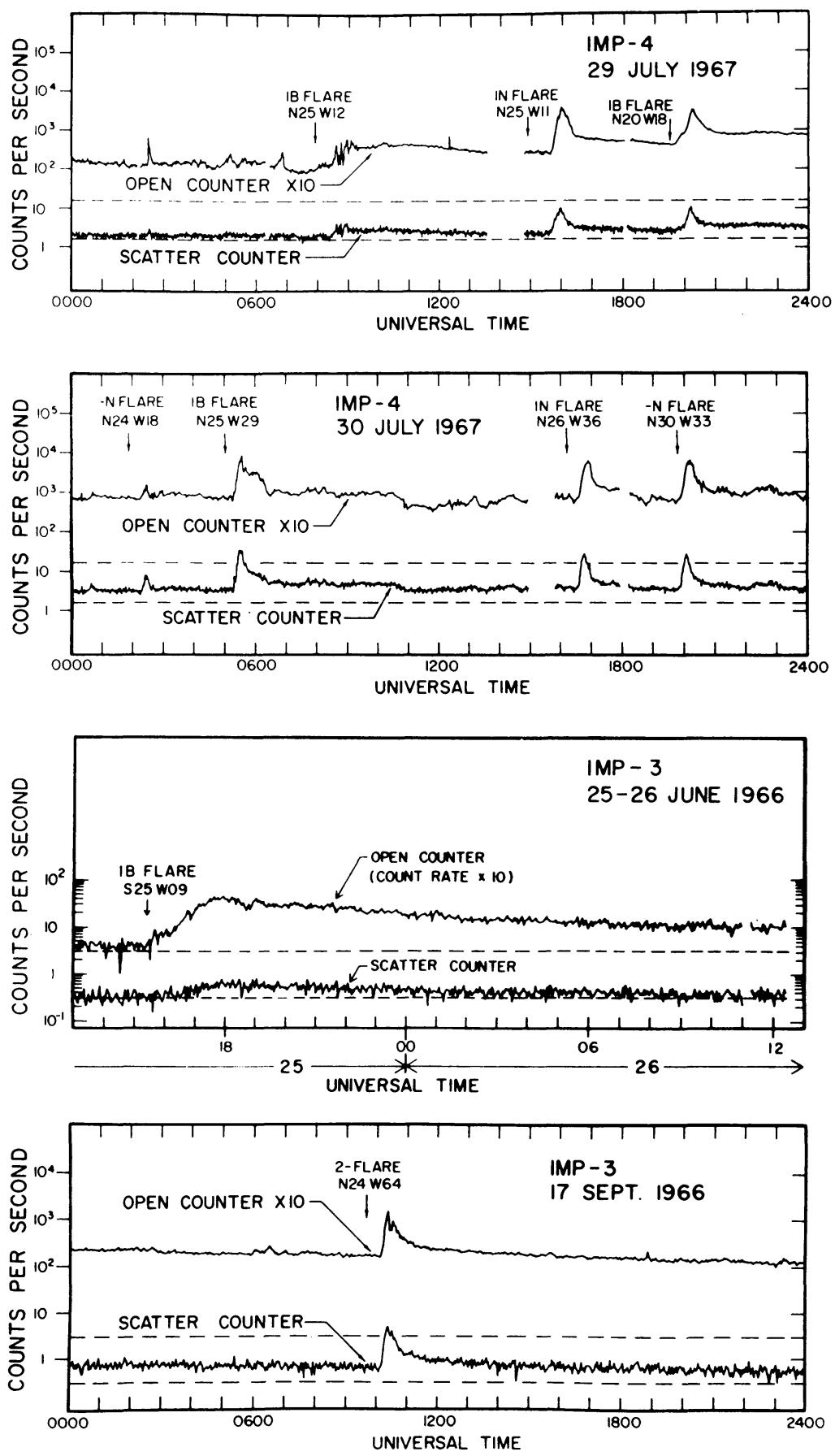

Fig. 1. Several examples of electron events observed by spacecraft at $1 \mathrm{AU}$ are shown here. The upper two panels show a series of rapid rise-rapid fall events, all from the same active region. The scatter counter is sensitive only to electrons above $45 \mathrm{keV}$ energy. 


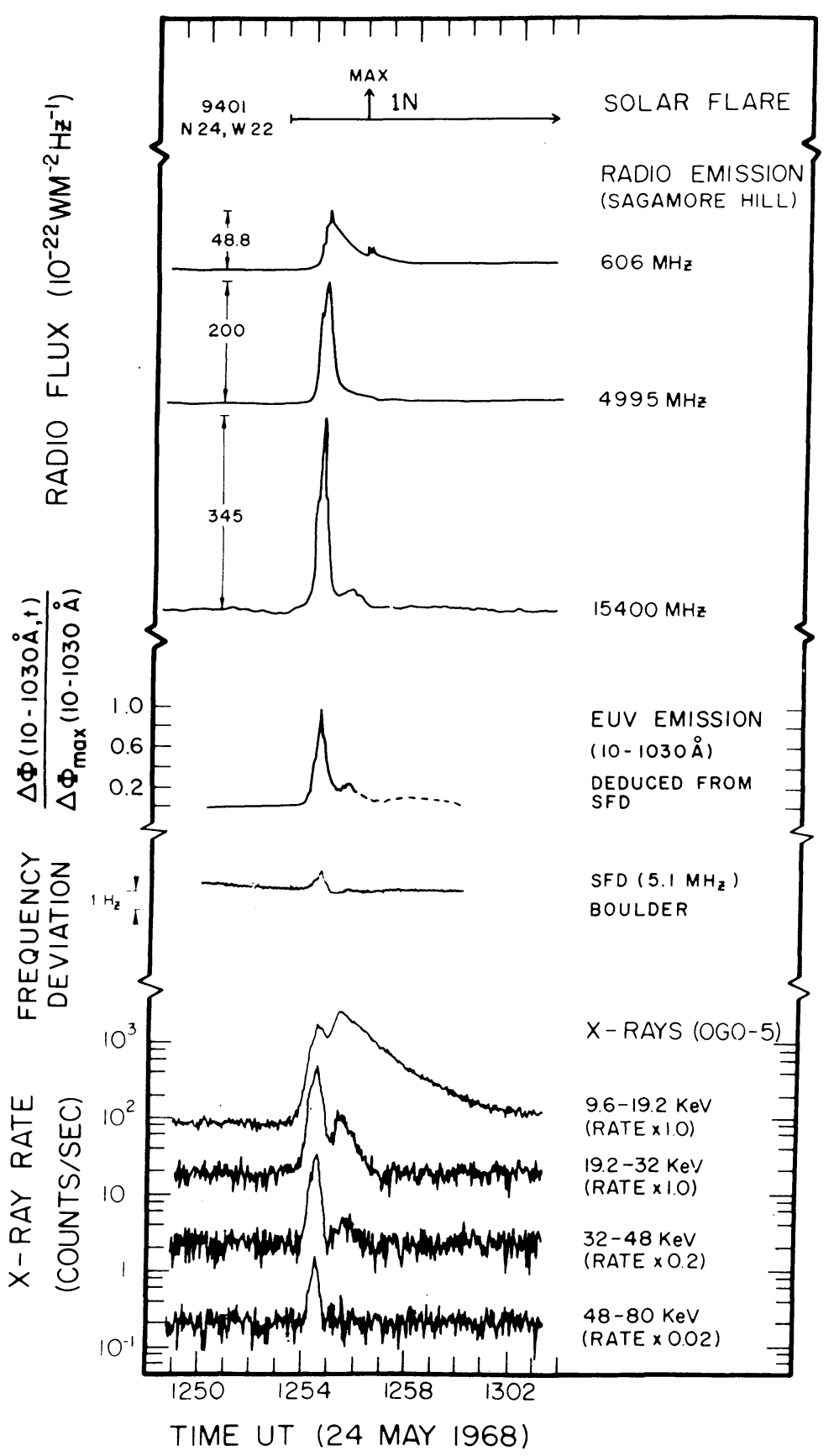

Fig. 2. An example of an impulsive non-thermal X-ray burst illustrating the close correspondence to the impulsive radio burst at $\mathrm{cm}$ wavelengths and EUV emission as derived from SFD measurements (Kane 1973b). 

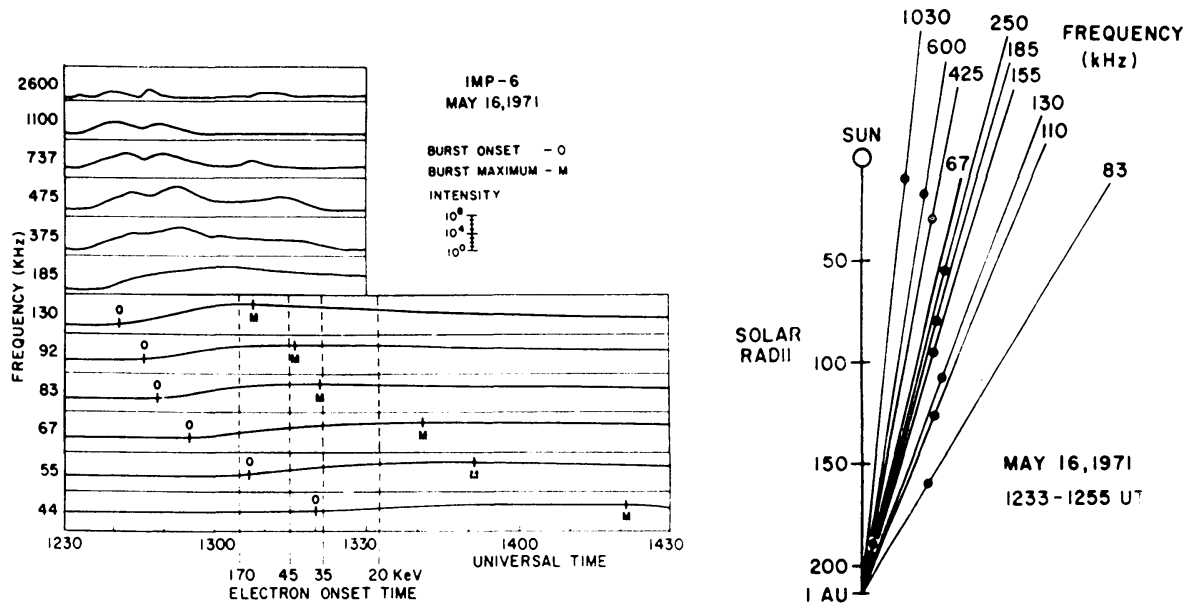

Fig. 3. The type III burst of 1971, May 16 observed at frequencies from $2.6 \mathrm{MHz}$ to $44 \mathrm{kHz}$. The $55 \mathrm{kHz}$ emission originates closest to $1 \mathrm{AU}$. The location of the radio emission at different frequencies, as derived from the modulation of the signal, is also shown.

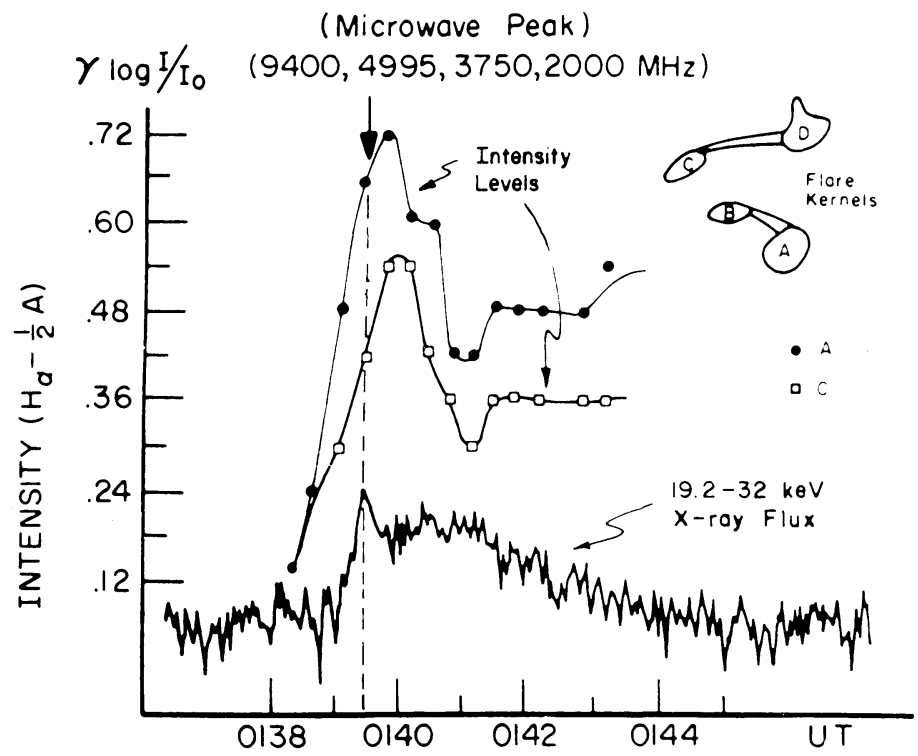

Fig. 4. Impulsive X-ray flux (19.2-32 keV) plotted along with the light curve measures at $\mathrm{Ha}-1 / 2 \AA$ during an impulsive flare. Of four potential bright points, only those labeled $A$ and $C$ exhibited the abrupt intensity increase associated with X-ray spikes. $I_{0}$ is the local background and $\gamma$, with a typical value of 3 , is the contrast of the film (from Vorpahl and Zirin, 1970).

The electromagnetic emissions observed from small electron flares are those typical of the flash phase of solar flares. Figure 5 shows a typical but idealized picture of these emissions and their timing. Not all of these types of emissions accompany every flare which produces electrons, but when such emissions occur the timing is usually as 


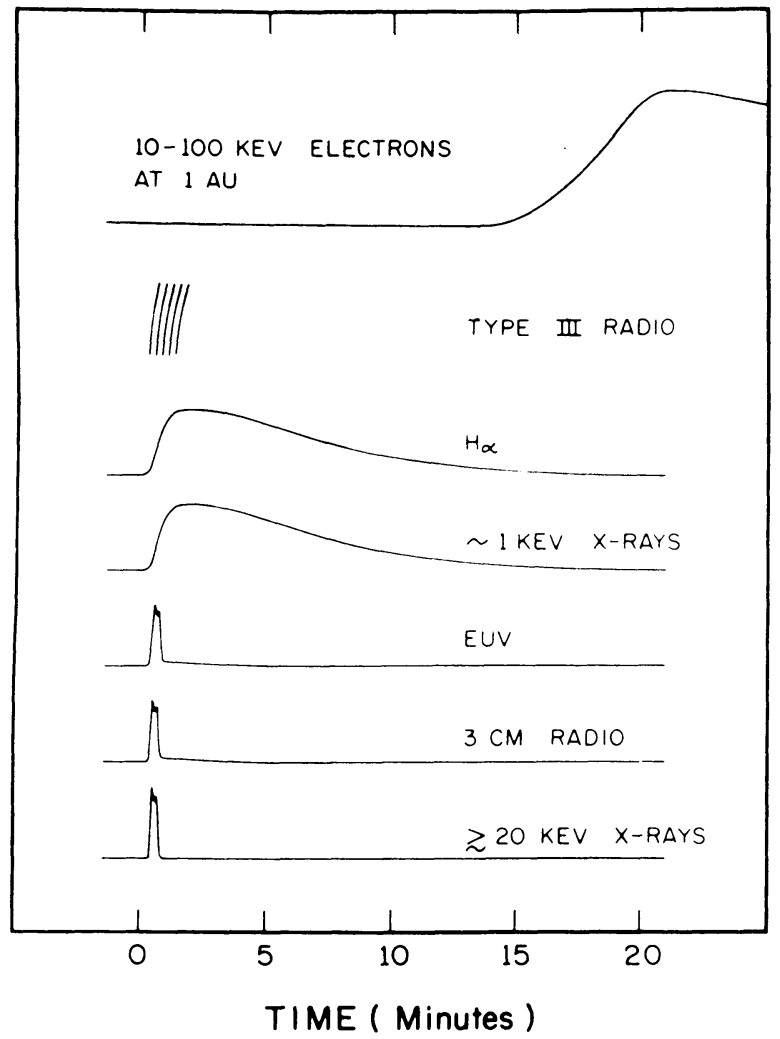

Fig. 5. An idealized picture of the flare flash phase event, showing the relative timing of each emission. Not all flare flash phases are accompanied by all these emissions but when they do occur, the timing is usually as shown.

shown. These emissions (and their appearance or non-appearance) can be interpreted as the direct consequence of the acceleration and subsequent motion of $10-100 \mathrm{keV}$ electrons in the solar atmosphere.

\section{Characteristics of Energetic Electrons at the Sun}

The most direct information about the energetic electrons in a flare is obtained from the non-thermal X-rays emitted by the flare. Energetic electrons at the Sun produce non-thermal $\mathrm{X}$-rays through bremsstrahlung in the solar atmosphere. Since the bremsstrahlung process is well understood and there is essentially no attenuation of these energy X-rays in the solar atmosphere, the observations of non-thermal X-ray bursts from the Sun can be directly and quantitatively interpreted in terms of energetic electrons at the Sun.

Microwave bursts and type III bursts are also observed in the radio range during the flash phase of solar flares (see review by Wild et al., 1963). These emissions, how- 


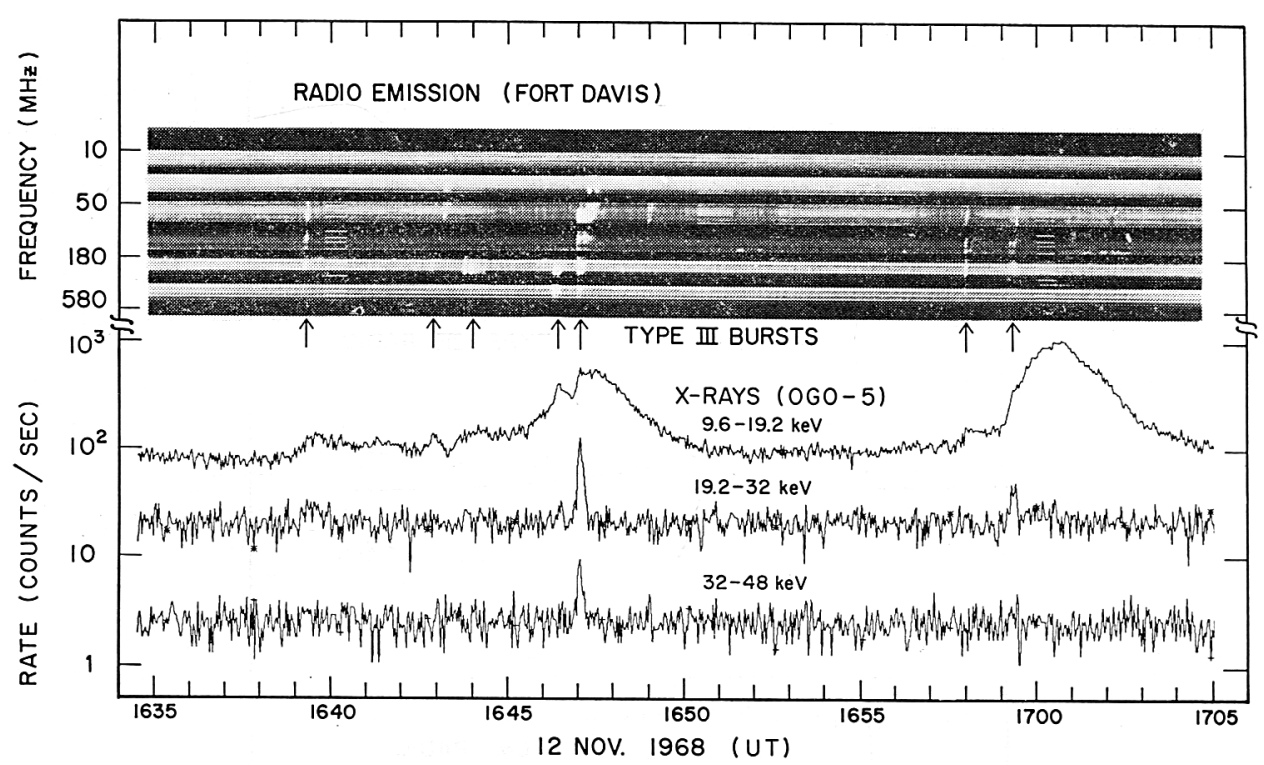

Fig. 6. Simultaneous X-ray and type III burst records, illustrating the close temporal correlation of the non-thermal X-ray bursts and type III bursts. (Kane, 1972).

ever, are much more difficult to interpret, due to substantial uncertainties in quantitatively evaluating the emission and propagation processes. However, the striking time coincidence and resemblance of microwave emission to non-thermal X-ray emission (Figure 2) (Anderson and Winckler, 1962; Arnoldy et al., 1968), and the time coincidence of type III bursts and X-ray bursts (Figure 6) leave little doubt that those radio emissions are due to the same electrons.

Several examples of $>10 \mathrm{keV}$ non-thermal $\mathrm{X}$-ray bursts during the flare flash phase are shown in Figures 2 and 6. Their properties are (Kane and Anderson, 1970):

(1) The duration of the burst is $\sim 10-100 \mathrm{~s}$ with $e$-folding rise and decay times of $2-10 \mathrm{~s}$. There is evidence that many X-ray bursts are actually composed of many spikes of $\sim$ few seconds duration. In fact the close temporal coincidence between type III bursts and X-ray bursts (see Figure 6) suggests that the acceleration time scales may be faster than $1 \mathrm{~s}$. The type III bursts typically occur in semi-periodic groups with individual bursts lasting $<1 \mathrm{~s}$ and burst to burst separation of $\lesssim$ few s. To the extent that can be observed with the available instrumental resolution, the $\mathrm{X}$-ray fine structure does correspond to type III radio burst group structure (S. R. Kane, 1974, private communication).

(2) The non-thermal burst typically occurs near the start of the soft X-ray burst (see Figures 2 and 6) although on most occasions a rise in soft X-rays is evident prior to the non-thermal burst. The non-thermal burst is usually coincident with the most rapidly rising portion of the soft X-ray profile.

(3) The energy spectrum of the photons between $\sim 10$ to $\sim 10^{2} \mathrm{keV}$ can be fitted to a power law, $\mathrm{d} J(h v) / \mathrm{d}(h v)=A(h v)^{-\gamma}$. A rapid falloff is often observed above $\sim 10^{2}$ 
$\mathrm{keV}$ when count rate statistics are sufficient at those energies. Such an X-ray spectrum would be produced by an electron population with power-law energy spectrum up to $\sim 100 \mathrm{keV}$ and cutoff beyond. Thermal spectra do not fit well to the data. Because the quasi-thermal component may mask observations below $\sim 10 \mathrm{keV}$, most observations of the non-thermal component have been limited to above $\sim 10 \mathrm{keV}$. However, the non-thermal component appears to extend to $\lesssim 5 \mathrm{keV}$ on those few occasions when it can be observed above the thermal component (Peterson et al., 1973; Kahler and Kreplin, 1971). The range of non-thermal X-ray spectra, illustrated in Figure 7, show
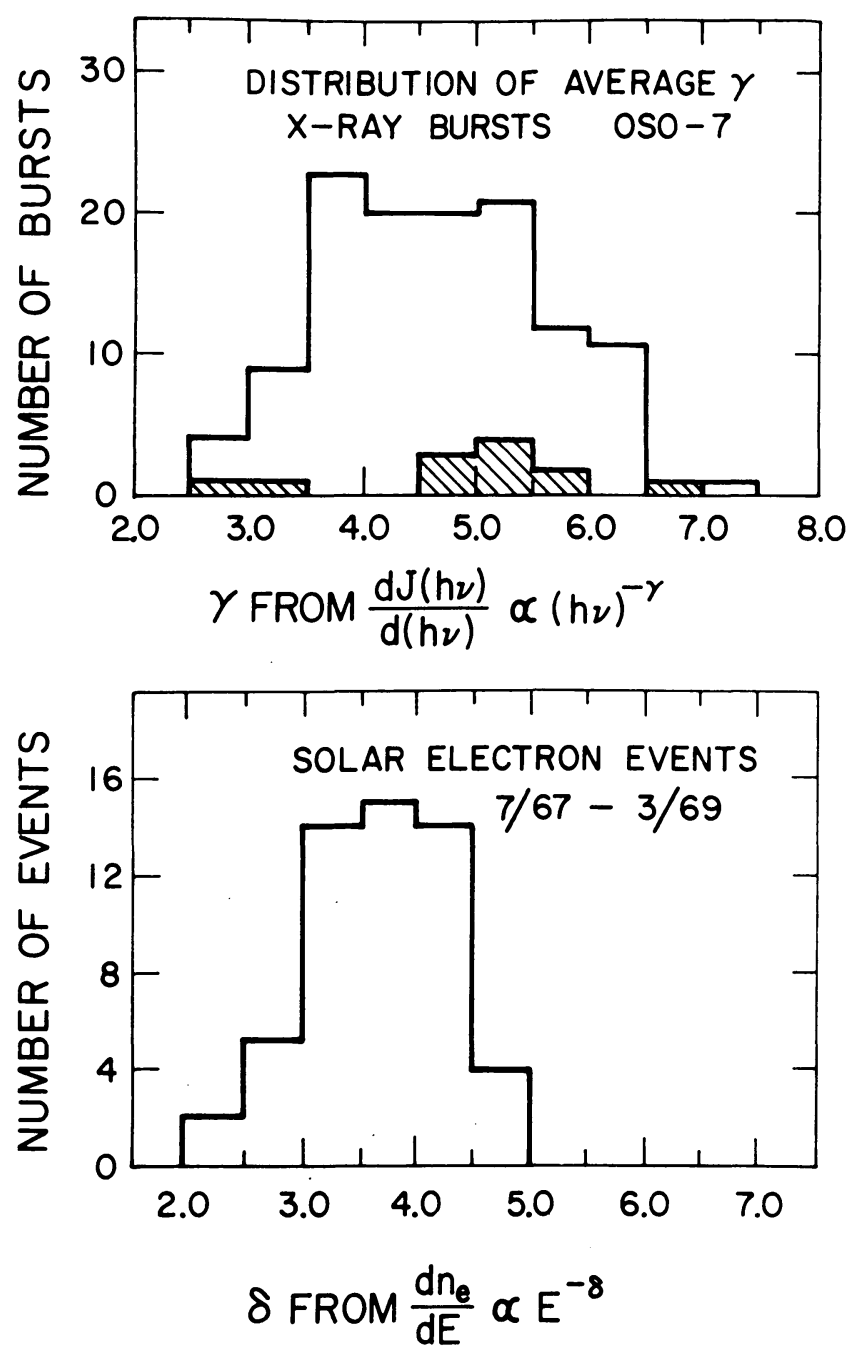

Fig. 7. The distribution of spectral exponents for non-thermal X-ray bursts (above) (from Datlowe, 1975) and electrons observed at $1 \mathrm{AU}$ (below). The shaded events in the X-ray histogram are those from importance 1 flares; the rest are from subflares. For the thin target model the electron exponent $\delta=\gamma-\frac{1}{2}$. 
a lower limit at $\gamma=3.0$ with very few bursts with harder spectra (Kane, 1971; Datlowe, 1975), and an upper limit of $\gamma \approx 6.5$. The limits $3.0 \lesssim \gamma \lesssim 6.5$ correspond to an instantaneous electron spectrum of $\mathrm{d} n / \mathrm{d} E \propto E^{-\delta}$ with $3.0 \lesssim \delta \lesssim 6.0$. Note the similarity to the range of electron spectra from interplanetary observations.

(4) The burst rise and decay times tend on many occasions to be smaller (more rapidly varying) for higher energies. Thus the X-ray spectrum and therefore the instantaneous electron spectrum varies from soft at burst onset ot hardest at burst maximum to soft again at burst end. This variation would be inconsistent with the simple model of impulsive injection of electrons, followed by decay of the electrons through collisional energy loss, because lower energy electrons would be lost most rapidly by collision, thus tending to harden the spectrum during the decay. Although more complex impulsive injection models (Brown, 1972) have been proposed to explain the energy variation during decay the most probable explanation is that the non-thermal electrons are being injected continuously over the period of the X-ray burst and the $\mathrm{X}$-ray energy variations just reflect the variations of the acceleration source.

(5) Non-thermal X-ray bursts are commonly observed in flares. Datlowe (1975) finds that $2 / 3$ of all flares observed by the OSO-7 soft X-ray detector were accompanied by non-thermal emission above $20 \mathrm{keV}$. The fact that the intensity of the non-thermal emission varies by 2-3 orders of magnitude from flare to flare within a given optical importance suggests that non-thermal electrons are present in every flare but may be too few in number in some flares to be observed.

\section{Relationship of Non-Thermal X-Ray Emission to Energetic Electrons}

The observed non-thermal X-rays can be related to the electrons at the Sun. Following Kane and Anderson (1970) we consider a volume $V$ in the solar atmosphere containing a relatively cold $\left(T \approx 10^{6}-10^{7} \mathrm{~K}\right)$ hydrogen plasma and energetic $(\gtrsim 10 \mathrm{keV})$ electrons. We assume the electron flux is isotropic; then the photon flux will also be isotropic. To obtain the X-ray flux at the Earth (distance $D=1 \mathrm{AU}$ ), let

$$
\begin{aligned}
& E \quad=\text { kinetic energy of an energetic electron }(\mathrm{keV}) \\
& h v \quad=\text { energy of an X-ray photon }(\mathrm{keV}) \\
& n_{\mathrm{i}} \quad=\text { number density of the hydrogen nuclei }\left(\mathrm{cm}^{-3}\right) \\
& \left.\frac{\mathrm{d} n(E)}{\mathrm{d} E}=\text { density of energetic electrons (electrons } \mathrm{cm}^{-3} \mathrm{~s}^{-1} \mathrm{keV}^{-1}\right) \\
& \frac{\mathrm{d} \sigma(E, \mathrm{~h} v)}{\mathrm{d}(h v)}=\text { differential cross section for electron-proton bremsstrahlung } \\
& \quad\left(\mathrm{cm}^{2} \text { ion }{ }^{-1} \mathrm{keV}^{-1}\right)
\end{aligned}
$$

For non-relativistic electrons $(E \leqslant 100 \mathrm{keV})$ the bremsstrahlung cross-section is 
approximately given by Bethe-Heitler formula (Jackson, 1962), written in our units $(E, h v$ in $\mathrm{keV})$ as

$$
\begin{aligned}
\frac{\mathrm{d} \sigma(E, h v)}{\mathrm{d}(h v)} & \approx 1.58 \times 10^{-24} \frac{1}{E h v} \ln \left[\left(\frac{E}{h v}\right)^{1 / 2}+\right. \\
& \left.+\left(\begin{array}{c}
E \\
h v
\end{array}-1\right)^{1 / 2}\right] \mathrm{cm}^{2} \text { ion }^{-1} \mathrm{keV}^{-1}
\end{aligned}
$$

Then at $1 \mathrm{AU}$ the differential spectrum of the X-rays produced in $V$ by bremsstrahlung is given by

$$
\begin{aligned}
\frac{\mathrm{d} J(h v)}{\mathrm{d}(h v)} & \approx 1.05 \times 10^{-42} \frac{n_{\mathrm{i}} V}{h v} \int_{h v}^{100} E^{1 / 2} \frac{\mathrm{d} n(E)}{\mathrm{d} E} \ln \left[\left(\begin{array}{c}
E \\
h v
\end{array}\right)^{1 / 2}+\right. \\
& \left.+\left(\begin{array}{c}
E \\
h v
\end{array}-1\right)^{1 / 2}\right] \mathrm{d} E \text { photons } \mathrm{cm}^{-2} \mathrm{~s}^{-1} \mathrm{keV}^{-1}
\end{aligned}
$$

Brown (1971) showed that this equation can be inverted for well-defined X-ray spectra to obtain the electron spectrum. For the special case of a power law-X-ray spectrum,

we obtain

$$
\mathrm{d} J / \mathrm{d}(h v)=A(h v)^{-v}
$$

$$
\frac{\mathrm{d} n}{\mathrm{~d} E}=1.21 \times 10^{42} \gamma(\gamma-1)^{2} B\left(\gamma-\frac{1}{2}, \frac{3}{2}\right) \frac{A E^{-\gamma+1 / 2}}{n_{\mathrm{i}} V},
$$

where $B(x, y)$ is the beta function. The electron energy spectrum is of the form $\mathrm{d} n / \mathrm{d} E \propto E^{-\delta}$ where $\delta=\gamma-\frac{1}{2} .^{*}$

The X-ray calculation also relates the X-ray intensity to the non-thermal 'emission measure', $n_{\mathrm{e}}\left(>E_{0}\right) n_{\mathrm{i}} V$, here defined with $n_{\mathrm{e}}\left(>E_{0}\right)=$ density of non-thermal electrons above energy $E_{0}, n_{\mathrm{i}}$ the ambient ion density in the $\mathrm{X}$-ray region and $V=$ volume of the $\mathrm{X}$-ray region. The important thing to note here is that these relationships hold at a given instant of time. That is, the instantaneous number and spectrum of energetic electrons in the X-ray region is related to the instantaneous X-ray emission. These relationships hold for the electron population in the $\mathrm{X}$-ray emitting region regardless of any assumptions about thick- or thin-target models. However, in order to obtain the total number and spectrum of electrons accelerated, the time each electron spends in the X-ray region (which may be a function of electron energy) and the ambient density, $n_{i}$, must be known.

Another important quantity which can be obtained independent of model is the minimum collisional energy loss of the electrons in the X-ray emitting region. This quantity can be obtained because both collisional and bremsstrahlung energy loss are

* This relationship has also been computed numerically with $\delta$ increasing sharply above $100 \mathrm{keV}$ (Kane and Anderson, 1970; Lin and Hudson, 1971), and gives $\gamma \approx \delta+0.7$ in the energy range $10 \mathrm{keV}<h v<80 \mathrm{keV}$. 
R.P. LIN

dependent on the ambient density so that the ratio of the two losses is independent of ambient density. For example, in fully ionized hydrogen, collision losses are (Trubnikov, 1965)

$$
\frac{\mathrm{d} E}{\mathrm{~d} t_{\text {collision }}}=-4.9 \times 10^{-9} n_{\mathrm{i}} E^{-1 / 2} \mathrm{keV} \mathrm{s}^{-1}
$$

and from Berger and Seltzer (1964) we obtain for bremsstrahlung

$$
\frac{\mathrm{d} E}{\mathrm{~d} t_{\text {brems }}}=-6.2 \times 10^{-18} n_{\mathrm{i}} E^{1 / 2}(E+988) \mathrm{keV} \mathrm{s}^{-1}
$$

so that the ratio is

$$
R(E)=\frac{\mathrm{d} E}{\mathrm{~d} t_{\text {brems }}}\left(\frac{\mathrm{d} E}{\mathrm{~d} t_{\text {collision }}}\right)^{-1}=1.27 \times 10^{-9} E(E+988)
$$

Figure 8 shows these ratios averaged over power law spectra from $\sim 20-100 \mathrm{keV}$ (Lin and Hudson, 1971) for both neutral and ionized hydrogen. By obtaining the total bremsstrahlung energy loss and multiplying by $R(E)$ one can obtain the minimum collisional energy loss in the $\mathrm{X}$-ray emitting region.

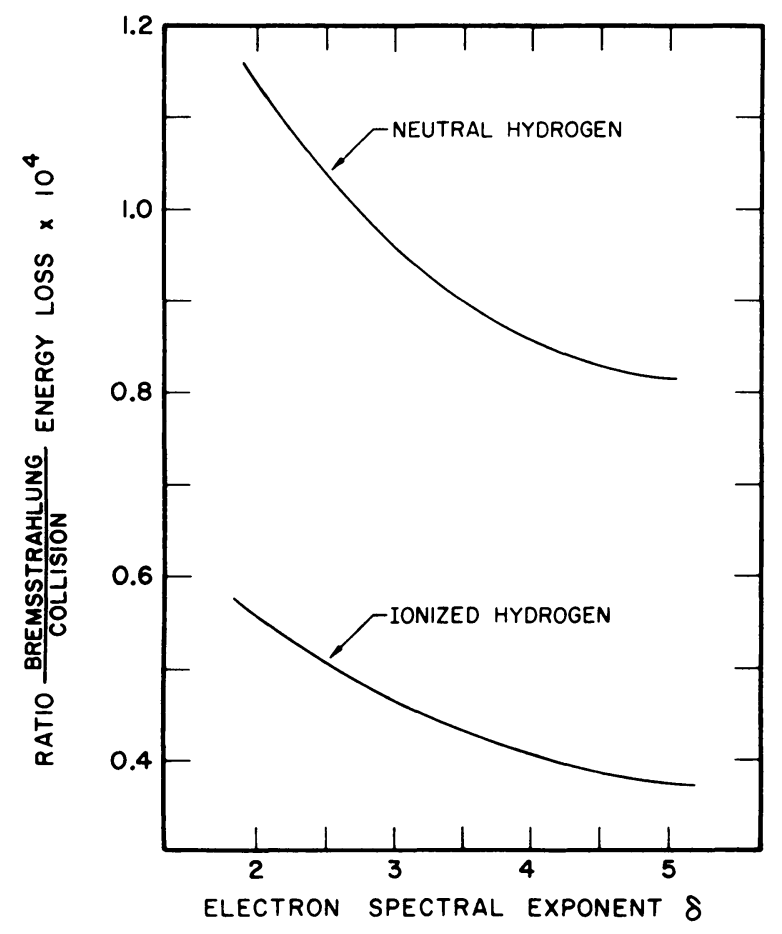

Fig. 8. The ratio of electron energy loss by bremsstrahlung to energy loss by collisions for both neutral and ionized hydrogen is shown here as a function of the electron spectral exponent. The energy losses are averaged over the electron spectrum in the energy range 22-100 keV. 


\section{The Evolution of the Electrons at the Sun}

The relationship of the instantaneous X-ray producing electron spectrum to the accelerated electron spectrum depends on the evolution of the electrons subsequent to acceleration. Suppose the electrons are accelerated in one region and produce the bulk of the observed X-rays in another region (these two regions may be one and the same but for the sake of generality we will allow them to be different). The evolution of the electron distribution, $N(E, t)=V(\mathrm{~d} n / \mathrm{d} E)$ where $V=$ volume in the X-ray emitting region, can be described by the equation

$$
\frac{\partial N(E, t)}{\partial t}=F(E, t)-\begin{gathered}
N(E, t) \\
\tau_{\mathrm{e}}(E)
\end{gathered}-\frac{\partial}{\partial E}\left[N(E, t) \frac{\mathrm{d} E}{\mathrm{~d} t}\right],
$$

where $F(E, t)$ is the input source of electrons $\mathrm{keV}^{-1} \mathrm{~s}^{-1}, N(E, t) / \tau_{\mathrm{e}}(E)$ is the number of electrons escaping the region per second, and the third term describes energy change processes for the electrons. These energy change processes could be loss by collisions with the ambient medium and/or loss by non-collisional processes such as wave-particle interactions. Although wave-particle interactions may be the dominant form of electron energy loss, the lack of relevant observations and the theoretical complexity of the interactions rule out the possibility of any quantitative estimates of their effects. Instead we shall treat just the collisional energy losses and bear in mind that these constitute a lower limit to the actual electron energy loss. Note that X-ray observations define $N(E, t)$ subject to a choice of ambient density $n_{\mathrm{i}}$ (Equation (4)). Thus this equation can be solved for $F(E, t)$, given $n_{\mathrm{i}}$ and given the form of $\tau_{\mathrm{e}}(E)$, if only collisional energy losses are assumed to be important. This solution has been carried out numerically for several X-ray bursts for various $n_{\mathrm{i}}$ and $\tau_{\mathrm{e}}(E)$ (Kane, 1973a).

To a good approximation we can consider $N(E, t)$ as constant over some time interval, $\Delta t$, and zero outside that interval. This removes the time dependence of the equation. Additionally we shall consider only the power-law case, $N(E, t)=B E^{-\delta}$, so that inserting for $\mathrm{d} E / \mathrm{d} t$ the energy loss in ionized hydrogen (Equation (5)) we obtain

$$
F(E)=N(E)\left[\frac{1}{\tau_{\mathrm{e}}(E)}+\frac{4.9 \times 10^{-9} n_{\mathrm{i}}\left(\delta+\frac{1}{2}\right)}{E^{3 / 2}}\right] .
$$

We have computed the anticipated energy dependence of $F(E, t)$ compared to $N(E, t)$ and $\mathrm{d} J(h v) / \mathrm{d}(h v)$, and the energy dependence of the escaping electrons for two extremes:

(1) where the escape term is much larger than the collisional energy loss term. This situation is the thin-target approximation for X-ray emission;

(2) where the collisional energy loss term is much larger than the escape term. This situation is the thick-target approximation for $\mathrm{X}$-ray emission.

We have used two obvious choices for the energy dependence of $\tau_{\mathrm{e}}$, although other forms might be appropriate. These two are: (1) $\tau_{\mathrm{e}}=$ constant, and (2) $\tau_{\mathrm{e}} \propto E^{-1 / 2}$, i.e., proportional to the scale size of the $\mathrm{X}$-ray region divided by the particle velocity. The results are summarized in Table $I$. 
The spectrum of electrons escaping from the X-ray region given in Table $\mathrm{I}$ is not necessarily the spectrum of the electrons escaping to the interplanetary medium. The electrons need not escape to the interplanetary medium to be lost from the X-ray region; they may also escape to the low density, $n_{\mathrm{i}} \lesssim 10^{9} \mathrm{~cm}^{-3}$, upper corona, where the flux of X-rays they produce will be below the threshold of current X-ray detectors. Also the acceleration region may be much higher in the solar atmosphere than the $\mathrm{X}$-ray region, and the electrons observed in space may have come directly from the accelerated population.

\section{TABLE I}

Spectral dependence of electrons and X-rays

\begin{tabular}{|c|c|c|}
\hline & Thick-target & Thin-target \\
\hline Spectrum of X-rays & $\frac{\mathrm{d} J(h v)}{\mathrm{d}(h v)}=-A(h v)^{-\gamma}$ & $\frac{\mathrm{d} J(h v)}{\mathrm{d}(h v)} A(h v)^{\prime}$ \\
\hline $\begin{array}{l}\text { Spectrum of electrons in } \\
\text { X-ray emitting region } \\
N(E) \propto \frac{\mathrm{d} n}{\mathrm{~d} E} \propto E^{-\delta}\end{array}$ & $\delta=\gamma-\frac{1}{2}$ & $\delta=\gamma-\frac{1}{2}$ \\
\hline $\begin{array}{l}\text { Spectrum of accelerated } \\
\text { electrons } F(E) \propto E^{-\delta_{a}}\end{array}$ & $\delta_{a}=\gamma+1$ & $\begin{array}{ll}\delta_{a} & \gamma-\frac{1}{2} \text { for } \tau_{\mathrm{e}} \text { constant } \\
\delta_{a} & \gamma-1 \text { for } \tau_{\mathrm{e}} x E^{-1 / 2}\end{array}$ \\
\hline $\begin{array}{l}\text { Spectrum of electrons } \\
\text { escaping from the X-ray } \\
\text { region, } S(E) \propto E^{-\delta_{e}}\end{array}$ & $\begin{array}{l}\delta_{\mathrm{e}}=\gamma-\frac{1}{2} \text { for } \tau_{\mathrm{e}}=\text { constant } \\
\delta_{\mathrm{e}}=\gamma-1 \text { for } \tau_{\mathrm{e}} \propto E^{-1 / 2}\end{array}$ & $\delta_{\mathrm{e}} \delta_{\mathrm{a}}=\left\{\begin{array}{l}\gamma-\frac{1}{2} \text { for } \tau_{\mathrm{e}}=\text { constant } \\
\gamma-1 \text { for } \tau_{\mathrm{e}} \propto E^{-1 / 2}\end{array}\right.$ \\
\hline
\end{tabular}

The time scale for loss of the electrons must be shorter than the most rapid decay observed for X-ray bursts. These decay time scales may be $\sim 1 \mathrm{~s}$ ( $e$-folding), faster than the resolution of current X-ray instrumentation. Similarly, the radio (type III) observations indicate the acceleration probably varies on a scale of $\lesssim 1 \mathrm{~s}$.

The collisional energy loss can be described in terms of a time constant

$$
\tau_{\mathrm{c}}=\frac{E^{3 / 2}}{4.9 \times 10^{-9}} \overline{\left(\delta+\frac{1}{2}\right) n_{\mathrm{i}}},
$$

where $E$ is in $\mathrm{keV}, n_{\mathrm{i}}$ in $\mathrm{cm}^{-3}$ (Equation (5)). For typical values of $\delta$, a collision time constant $\tau_{\mathrm{e}} \approx 1 \mathrm{~s}$ implies $n_{\mathrm{i}} \approx 6 \times 10^{10} \mathrm{~cm}^{-3}$ for $E=100 \mathrm{keV} ; n_{\mathrm{i}} \approx 10^{10} \mathrm{~cm}^{-3}$ for $E \approx 50$ $\mathrm{keV}$ and $n_{\mathrm{i}} \approx 2 \times 10^{9} \mathrm{~cm}^{-3}$ for $E=10 \mathrm{keV}$.

On the other hand $\tau_{\mathrm{e}}$ may be as short as the rectilinear travel time to cross the X-ray emitting region. This region is likely to be a few thousand $\mathrm{km}$ thick to that $\tau_{\mathrm{e}} \gtrsim$ $0.02-0.1 \mathrm{~s}$ for $\sim 5-100 \mathrm{keV}$ electrons.

\section{Thick- or Thin-Target?}

Datlowe and Lin (1973) noted that it is possible to distinguish between thick- and thin-target cases under the assumption that the spectrum of electrons observed in the interplanetary medium is representative of the accelerated electron spectrum (i.e., 

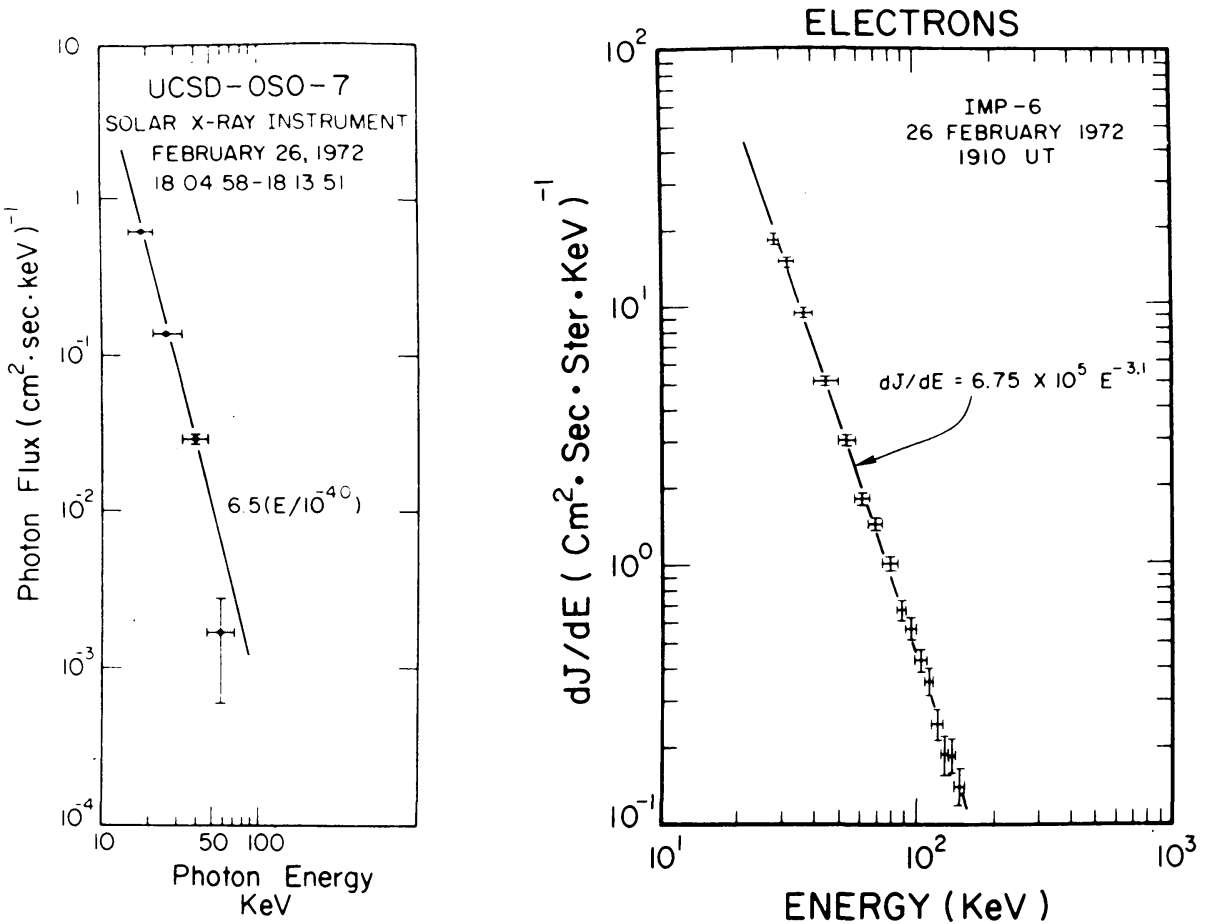

Fig. 9. The spectra of hard X-rays and electrons observed at $1 \mathrm{AU}$ for the same flare event. The photons fit a power law spectrum $\mathrm{d} J(h v)=A(h v)^{-\gamma}$ where $\gamma=4.0 \pm 0.3$, while the electrons fit a spectrum $\mathrm{d} J / \mathrm{d} E=6.75 \times 10^{5} E^{-3.1}$. Since $\mathrm{d} n / \mathrm{d} E=v \mathrm{~d} J / \mathrm{d} E$ where $v$ is the electron velocity, the electron fit a density spectrum $\mathrm{d} n / \mathrm{d} E \propto E^{-\delta}$ with $\delta=3.6 \pm 0.1$. These two spectra are consistent with thin target emission under the assumption the escaping electrons have the same spectrum as the accelerated electrons.

$\delta_{\mathrm{e}}=\delta_{\mathrm{a}}$, see Table I). For one flare event where high energy resolution measurements were available for both the electrons and X-rays above $20 \mathrm{keV}$ (Figure 9), the result was $\delta_{\mathrm{a}}=\gamma-\frac{1}{2}$, favoring thin-target. Other $\mathrm{X}$-ray electron events studied where only measurements with poor energy resolution were available are also generally consistent (see Lin and Hudson, 1971; and Kane and Lin, 1972) with a thin-target model. The thin-target case is also consistent with the location of the acceleration region $\left(n_{\mathrm{i}} \lesssim 10^{10} \mathrm{~cm}^{-3}\right)$ derived from considerations of the low energy electron spectrum observed at $1 \mathrm{AU}$.

In favor of thick-target processes we note that if non-relativistic electrons penetrate to the dense $\left(n_{\mathrm{i}} \gtrsim 10^{12} \mathrm{~cm}^{-3}\right)$ regions of the chromosphere-corona boundary and below, they could produce the observed EUV and peraps provide the energy for heating the $\mathrm{H} \alpha$ flare region through collisional loss (and possibly even heating the white light flare region) (Hudson, 1972). The close time coincidence between the hard X-ray spike and the EUV spike (Kane and Donnelly, 1971) is consistent with such an interpretation. At those densities the thick-target approximation would certainly be appropriate. 
There are several possibly ways of reconciling the observations in support of thickand thin-target. One possibility is that the escape of the electrons from the flare is highly energy dependent so that the spectrum observed in the interplanetary medium is not representative of the accelerated electron spectrum.

A second possibility is that electrons of low energies, say below $\sim 10 \mathrm{keV}$, are described by the thick-target approximation while higher energy electrons are in essentially thin-target situation (Kane, 1973a). This dichotomy could arise, for example, if the electrons are accelerated and contained by a magnetic 'bottle' in a low density, $n_{\mathrm{i}} \lesssim 10^{10} \mathrm{~cm}^{-3}$, region. Electrons only appear in high density, $n_{\mathrm{i}} \lesssim 10^{10} \mathrm{~cm}^{-3}$, regions near the feet of the magnetic bottle if they are scattered into the loss cone. Since the amount of scattering is a strongly decreasing function of energy, essentially only the low energy electrons will be dumped into the loss cone. This interpretation is consistent with the observations which show that the correspondence between rising portion of the EUV emission and the rising portion of the non-thermal X-rays is best for the lowest energy, $\sim 10 \mathrm{keV}, \mathrm{X}$-rays.

\section{Energy in Non-Relativistic Electrons}

The total energy contained in energetic electrons in these small solar flares is obtained by summing losses from the various processes which the electrons undergo, including collision loss, bremsstrahlung and gyro-synchrotron emission, and escape into the interplanetary medium. Almost a dozen events with both impulsive hard X-ray bursts and electrons subsequently observed at $1 \mathrm{AU}$ have been analyzed (Lin and Hudson, 1971; Kane and Lin, 1972) to obtain the energy in non-relativistic electrons. In addition, Datlowe (1975) has analyzed over 100 non-thermal X-ray burst events from subflares and importance 1 flares. Typical energy values are given in Table II.

\section{(a) COLLISIONAL ENERGY LOSS}

An important difference between thick- and thin-target for flare processes lies in the amount of energy lost by collisions of the non-thermal electrons to the ambient medium. This collision energy loss is the non-thermal electron energy input available to support other flare processes such as heating of the quasi-thermal X-ray plasma and production of EUV and $\mathrm{H} \alpha$ radiation. The thin-target approximation clearly will give a lower limit to the non-thermal electron collisional energy loss, while the thick-target approximation represents an upper limit. These limits on the collision energy loss from electrons above energy $E_{0}$ can be computed from the observed X-ray emission. Suppose

$$
\frac{\mathrm{d} J(h v)}{\mathrm{d}(h v)}=A(h v)^{-\gamma}
$$

then the rate of collisional energy loss is

$$
P_{\text {thin }}\left(>E_{0}\right)=9.4 \times 10^{24} A \gamma(\gamma-1) B\left(\gamma-\frac{1}{2}, \frac{3}{2}\right) E_{0}^{-(;-1)} \mathrm{erg} \mathrm{s}^{-1}
$$


TABLE II

Energy balance for a small electron flare

\begin{tabular}{ll} 
Energy in erg & \\
\hdashline $5 \mathrm{keV}$ & $>20 \mathrm{keV}$
\end{tabular}

A. Non-thermal electron energy

1. Electron energy loss through collisions
(a) Thin-target
$\sim 10^{30}$
$\sim 5 \times 10^{28}$
(b) Thick-target
$\sim 4 \times 10^{30}$
$\sim 2 \times 10^{29}$

2. Other electron energy loss processes bremsstrahlung X-ray emission gyro-synchrotron radio emission escape to the interplanetary medium

$\lesssim 0.01$ of thin-target collision loss

3. Total Energy in Accelerated Electrons
(a) Thin-target
(b) Thick-target
$\sim 5 \times 10^{30}$
$\sim 3 \times 10^{29}$
$\sim 5 \times 10^{30}$
$\sim 2 \times 10^{29}$

B. Quasi-thermal flare energy
1. Soft X-ray plasma
$\sim 5 \times 10^{29} \mathrm{erg}$
2. EUV emission
$\sim 5 \times 10^{28} \mathrm{erg}$
3. $\mathrm{H} x$ and other optical emissions
$\sim 10^{28} \mathrm{erg}$

for thin-target, ${ }^{*}$ and

$$
P_{\text {thick }}\left(>E_{0}\right)=9.4 \times 10^{24} A \gamma(\gamma-1) B\left(\gamma-\frac{1}{2}, \frac{3}{2}\right) E_{0}^{-(\gamma-1)} \mathrm{erg} \mathrm{s}^{-1}
$$

for thick-target,* so that the ratio

$$
\left(P_{\text {thick }} / P_{\text {thin }}\right)=\gamma \text {. }
$$

Note that this ratio is independent of the low energy cut-off $E_{0}$.

The range of $\gamma$ is observed to be from $\sim 2$ to $\sim 7$. Typical values of the collision energy loss for the small electron flares are given in Table II for both thick- and thintarget approximations.

\section{(b) ENERGY IN ELECTRONS ESCAPING TO THE INTERPLANETARY MEDIUM}

The energy lost in escaping electrons can be obtained by estimating the number of escaping electrons using simple propagation theory (Lin and Hudson, 1971) and multiplying by the average electron energy. Typical values for the energy lost in escaping electrons are $10^{-2}$ to $10^{-3}$ the collision energy loss, even in the thin-target case (Lin and Hudson, 1971), for flares where the energetic electrons contain the bulk of the energy. Thus, only about 0.1 to $1 \%$ of the electrons escape to the interplanetary medium in those cases.

* These expressions differ from what would be derived from Brown's (1971) expressions. The difference is due to a different expression for $\mathrm{d} E / \mathrm{d} t$ and some numerical errors in Brown (1971). 
(c) ENERGY LOST IN RADIO EMISSION

Another electron energy loss mechanism is gyro-synchrotron emission in the solar magnetic field. Gyro-synchrotron emission is the most likely explanation for the impulsive microwave burst. Takakura (1972) notes that careful consideration of a realistic model taking into account the non-uniformity of the magnetic field, and the selfabsorption of the radio emission indicates that the electron population producing hard X-rays will also produce the microwave burst with the observed spectrum and intensity. Since these electrons are not relativistic, the Larmor formula for the power radiated (Jackson, 1962) per electron is appropriate and leads to

$$
P \approx 3 \times 10^{-9} B_{\perp}^{2}{E \mathrm{~s}^{-1}}^{-1}
$$

where $B$ is the magnetic field in gauss and $E$ is the electron energy in keV. For choices of $B$ and $n_{\mathrm{i}}$ appropriate for the emission under this model we find that the collision loss is $\gtrsim 10^{2}-10^{3}$ times the loss by gyro-synchrotron emission for $10-100 \mathrm{keV}$ electrons.

Type II radio bursts are produced through the excitation of plasma waves by a coherent Cerenkov mechanism (see next section). These non-collisional wave-particle interactions, although they are a negligible energy loss mechanism at $1 \mathrm{AU}$ (Lin et al., 1973), may be a substantial electron energy loss process nearer the Sun.

\section{(d) TOTAL ENERGY IN ACCELERATED ELECTRONS}

Under the thin-target assumption the fraction of electrons lost in collisions must be small. Therefore the collisional energy loss given by Table II for the thin-target case is only a small fraction of the total energy in accelerated electrons. The bulk of the accelerated electrons under the thin-target assumption must escape from the dense regions where they would be lost by collisions. Escape to the interplanetary medium is rather negligible. However, as mentioned earlier the electrons may also escape to the low density, $n_{\mathrm{i}} \lesssim 10^{9} \mathrm{~cm}^{-3}$ upper corona, where the flux of X-rays they produce will be below the thresholds of current X-ray detectors.

We have, therefore, estimated the energy contained in accelerated electrons in the thin-target case by assuming that the electron lifetime ( $\tau_{\mathrm{e}}$ in this case) in the X-ray producing region is $\lesssim 1 \mathrm{~s}$. This time scale would be consistent with the observed variations of the $\mathrm{X}$-ray bursts.

The thick-target collisional energy loss should correspond closely to the total accelerated electron energy since the thick-target assumption implies that the bulk of the electrons is lost through collisions.

Two low energy cutoffs $\left(E_{0}\right)$ have been used in these computations: $20 \mathrm{keV}$ because most of the hard X-ray and electron measurements have been made above that energy; and $5 \mathrm{keV}$ since the few observations available of electrons and X-rays at low energies indicate that the non-thermal spectrum commonly extends to that energy. The energy in accelerated electrons above $\sim 20 \mathrm{keV}$ already constitutes a large fraction of the total flare energy. 


\section{(e) NUMBERS OF ELECTRONS}

The total number of electrons accelerated can be obtained by dividing the total energy in accelerated electrons by the average electron energy. This method yields $\sim 10^{38}$ electrons above $\sim 5 \mathrm{keV}$, or $\sim 10^{36}$ above $\sim 20 \mathrm{keV}$. The number escaping to the interplanetary medium is $\sim 10^{33}$ above $20 \mathrm{keV} \sim$ or $\sim 0.1 \%$ of the total number accelerated. This compares with estimates of Holt and Ramaty (1969) for the escape efficiency of relativistic electrons of between $0.6 \%$ to $23 \%$.

If the $>20 \mathrm{keV} \mathrm{X}$-ray production is assumed to occur at an ambient density of $\gtrsim 10^{10} \mathrm{~cm}^{-3}$ then the maximum instantaneous number of $>20 \mathrm{keV}$ electrons in the $\mathrm{X}$-ray region is estimated to be $\lesssim 10^{35}$, or at least one order of magnitude fewer than the total number accelerated. This confirms that the acceleration of electrons must have occurred continually over the duration of the X-ray burst.

\section{Total Energy in a Small Electron Flare}

Sufficient observations are now available so that an essentially complete energy balance for a small solar electron flare can be constructed. For comparison this energy balance is also given in Table II.

\section{(a) SOFT X-RAYS}

Essentially all flares are observed to be accompanied by soft X-ray emission. The hard impulsive $\mathrm{X}$-ray component appears to coincide with the most rapidly increasing portion of the soft X-rays' rise, as if the $10-100 \mathrm{keV}$ electrons were giving up their energy to heating up the soft X-ray producing plasma (Kahler et al., 1970; McKenzie et al. 1973). Although some heating of the soft X-ray plasma is probably provided by the $10-100 \mathrm{keV}$ electrons it is unlikely that the entire soft X-ray burst is due to this process because (1) the soft X-ray burst is observed in many flares without any substantial hard impulsive burst; and (2) the soft X-ray emission appears to begin before the hard impulsive X-ray bursts and continues to rise after the end of the hard X-ray emission (Datlowe, 1975).

Measurements of the soft X-ray spectrum provide the temperature. $T$, and emission measure, $n_{\mathrm{e}} n_{\mathrm{i}} V$, of the soft $\mathrm{X}$-ray plasma. Thus, the observations are able to define unambiguously the quantity (since $n_{\mathrm{e}} \approx n_{\mathrm{i}}$ for the 5 to $30 \times 10^{6} \mathrm{~K}$ flare plasmas)

$$
n_{\mathrm{e}} U=3 k T n_{\mathrm{e}} n_{\mathrm{i}} V
$$

A typical emission measure for these small flares is $n_{\mathrm{e}} n_{\mathrm{i}} V \approx 10^{48} \mathrm{~cm}^{3}$ and typical temperature is $T \sim 10^{7} \mathrm{~K}$ (Hudson et al., 1969; McKenzie et al., 1973), so that $n_{\mathrm{e}} U \approx 5 \times 10^{39} \mathrm{erg} \mathrm{cm}^{-3}$.

The volume of the soft $X$-ray region for a small flare is estimated to be $\sim 10^{28} \mathrm{~cm}^{-3}$ from measurements of the X-ray source size (Krieger et al., 1972; Thomas and Neupert, 1971), so that the density $n_{\mathrm{e}} \approx 10^{10}$. The energy in the soft X-ray plasma is then 
$\sim 5 \times 10^{29} \mathrm{erg}$. In view of the approximations, this number is an order of magnitude estimate.

(b) EUV EMISSION

EUV emission in the wavelength range 10 to $1040 \AA$ is commonly observed coinciding with and showing similar structure to the impulsive hard X-ray burst (Figure 2). The total energy emitted in EUV appears proportional to the energy in bremsstrahlung $\mathrm{X}$-rays with a factor (Kane and Donnelly, 1971)

$$
\varepsilon_{\mathrm{EUV}}(10-1030 \AA) \approx 5 \times 10^{4} \varepsilon_{\mathrm{X} \text { ray }}(10-50 \mathrm{keV}) .
$$

For small electron flares a typical $\varepsilon_{\mathrm{EUV}}$ is $\sim 5 \times 10^{28} \mathrm{erg}$. Kane and Donnelly (1971) noted that the best agreement is found between the EUV and X-rays of $\sim 10 \mathrm{keV}$ energy.

\section{(c) $\mathrm{H} \alpha$ EMISSION}

The total $\mathrm{H} \alpha$ emission energy can be estimated from the characteristics of the average importance 1 flare (Smith and Smith, 1963). The area is taken as $\sim 100$ millionths of the disk; the maximum bandwidth, $\sim 3 \AA$, the peak intensity, $\sim 0.8$ of the continuum level, and the duration, $\sim 10^{3} \mathrm{~s}$. The average emission in $\mathrm{H} \alpha$ is therefore $\sim 10^{28} \mathrm{erg}$.

Smith and Smith (1963) note that a significant fraction of the visible spectrum energy of a flare is contained in $\mathrm{H} \alpha$, particularly for small flares. Energetically the optical emission constitutes a very minor part of an electron flare.

\section{(d) TOTAL ENERGY IN FLARE}

The energy in each part of the flare is listed in Table II. We have not included mechanical energy from mass motion in the flare or any dissipation/cooling which does not result in observable radiation. For the large flares which produce interplanetary shocks the energy contained in the shock is a major and sometimes dominant part of the total flare energy budget (Hundhausen, 1972). However, for these small flares no signs of shock phenomena are observed, either in the radio emission or in interplanetary space.

It is quite clear from Table II that the bulk of the energy in a small electron flare resides in the non-thermal electron population. In the thin-target cases the collisional energy loss of the $>5 \mathrm{keV}$ electrons is of the same order as the energy needed to produce the other flare emission. Under the thick-target approximation too much energy, by about an order of magnitude, is available from the electrons.

\section{Location of the Electron Acceleration Region}

Measurements of the electron energy spectrum observed at $1 \mathrm{AU}$ down to low energies can be interpreted in terms of an upper limit to the amount of material traversed by the accelerated particles (Lin, 1973). Suppose the electrons are accelerated at a height, $h$, in the solar atmosphere, and then pass through the overlying material (fully ionized 
hydrogen) to reach the vicinity of 1 AU. Using Trubnikov's (1965) expression for energy loss in ionized hydrogen (Equation (5)) we obtain for non-relativistic electrons

$$
\mathrm{d} E=-2.6 \times 10^{-18}{ }^{n_{\mathrm{i}}} \mathrm{keV} \mathrm{cm}^{-1} \text {. }
$$

Integrating from height $h$ to $1 \mathrm{AU}$

$$
\begin{aligned}
& \int_{E_{1}}^{E_{2}} E \mathrm{~d} E=-2.6 \times 10^{-18} \int_{h}^{1 \mathrm{AU}} n_{\mathrm{i}}(x) \mathrm{d} x \equiv K(h), \quad \text { and } \\
& E_{2}=\left(E_{1}^{2}-2 K\right)^{1 / 2},
\end{aligned}
$$

where $E_{1}$ is in initial accelerated energy of the electron and $E_{2}$ its energy at $1 \mathrm{AU}$. If the spectrum of the freshly accelerated electrons at the Sun is given by a power law in energy, as would be consistent with the X-ray observations,

$$
\frac{\mathrm{d} n}{\mathrm{~d} E_{1}}=A E_{1}^{-\delta}
$$

then the spectrum of the electrons observed at $1 \mathrm{AU}$ will be

$$
\frac{\mathrm{d} n}{\mathrm{~d} E_{2}}=\frac{A E_{2}}{\left(E_{2}^{2}+2 K\right)^{(\delta+1) / 2}} .
$$

This spectrum has a maximum at

$$
E_{2 M}=\left(\begin{array}{c}
2 K \\
\delta
\end{array}\right)^{1 / 2}
$$

Actually for a given height $h$, the location of the maximum must be above this energy because:

(i) the direct radial distance outward through the solar atmosphere is used in the calculation without taking into account the helical paths followed by the particles;

(ii) scatterings which change the particle's direction are far more effective for low energy particles than for high energy particles and will subject the low energy particles to longer path lengths;

(iii) no other energy loss mechanisms such as generation of radio or plasma waves, etc., are taken into account.

None of the low energy solar electron spectra observed to date show a turnover above $\sim 6 \mathrm{keV}$ (Figure 10). Thus

$$
\int_{h}^{1 \mathrm{AU}} n_{\mathrm{i}}(x) \mathrm{d} x \leqslant 3.5 \times 10^{19} \mathrm{~cm}^{-2} .
$$



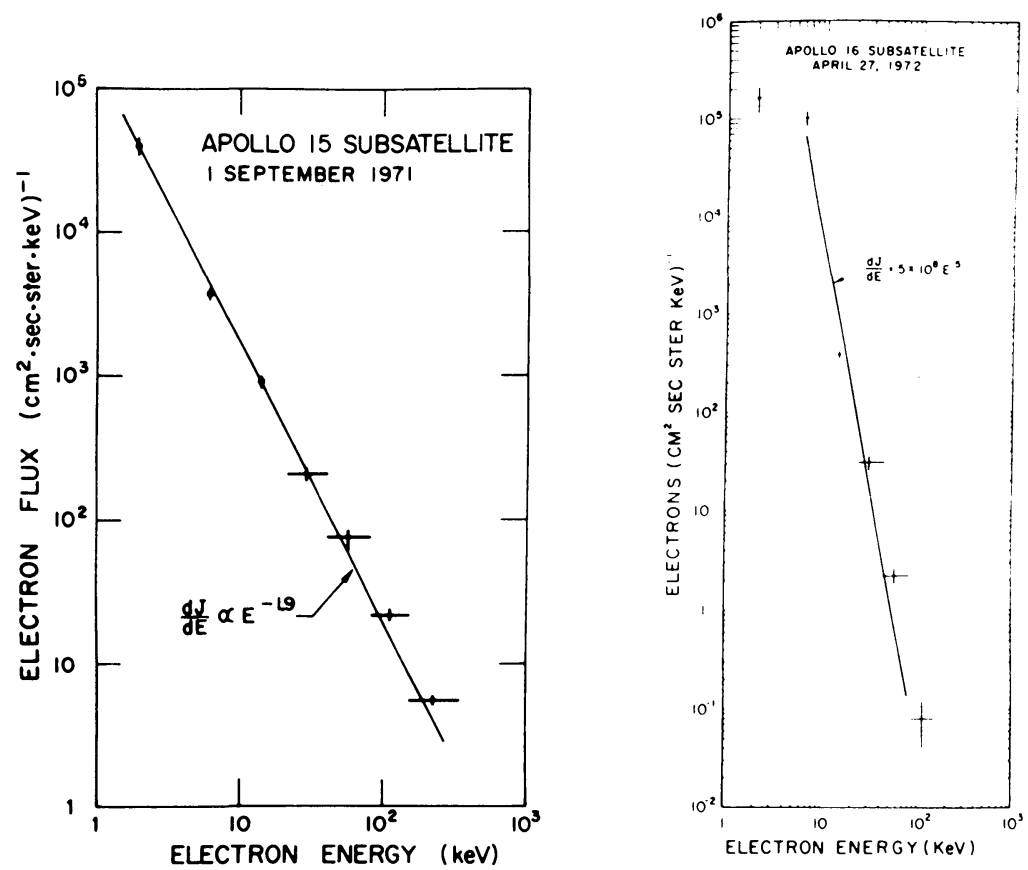

Fig. 10. Two electron energy spectra extending to low energies. The September 1 event is accompanied by energetic protons while the 27 April event (from Lin et al., 1973) is not. Both spectra extend smoothly in a power law to below $\sim 6 \mathrm{keV}$.

We wish to re-emphasize the fact that this estimate is a lower limit to the actual height of acceleration since the effects which were not taken into account would tend to increase the minimum energy of the peak. Clearly the electron acceleration must have occurred in the transition region or lower corona. Although only a few events have been observed to energies below $\sim 20 \mathrm{keV}$, in no events has a turnover been observed at higher energies. Thus electron acceleration at the flash phase appears to be a coronal phenomenon, at least for events observed to emit electrons into the interplanetary medium.

This location and ambient density is consistent with the observed starting frequencies $(\sim 200-1000 \mathrm{MHz})$ of type III bursts, and is also consistent with the occasional observation of an electron event at $1 \mathrm{AU}$ without detectable X-ray emission (Kane and Lin, 1972). Presumably in those events the magnetic field structure in the vicinity of the acceleration region is such as to prevent the electrons from entering dense regions where a detectable flux of X-rays would be produced.

\section{Flash Phase Proton Acceleration}

One question of importance in distinguishing possible flare particle acceleration mechanisms during the flash phase is whether or not protons as well as electrons are 
accelerated, and if so whether the acceleration mechanism accelerates particles to the same energy or the same velocity of rigidity, and what are the relative efficiencies for electrons and protons (Syrovatskii, 1969). Recently McDonald et al. (1972) noted that on occasion proton micro-events are observed. These events are characterized by extremely low fluxes, $\sim 10^{-2}\left(\mathrm{~cm}^{2} \mathrm{~s} \mathrm{sr}\right)^{-1}$ above $10 \mathrm{MeV}$ which, however, are observed up to $\gtrsim 50 \mathrm{MeV}$, and no indications (such as type II and IV emission) of a second phase in the associated flare phenomena. We have plotted the maximum $>45 \mathrm{keV}$

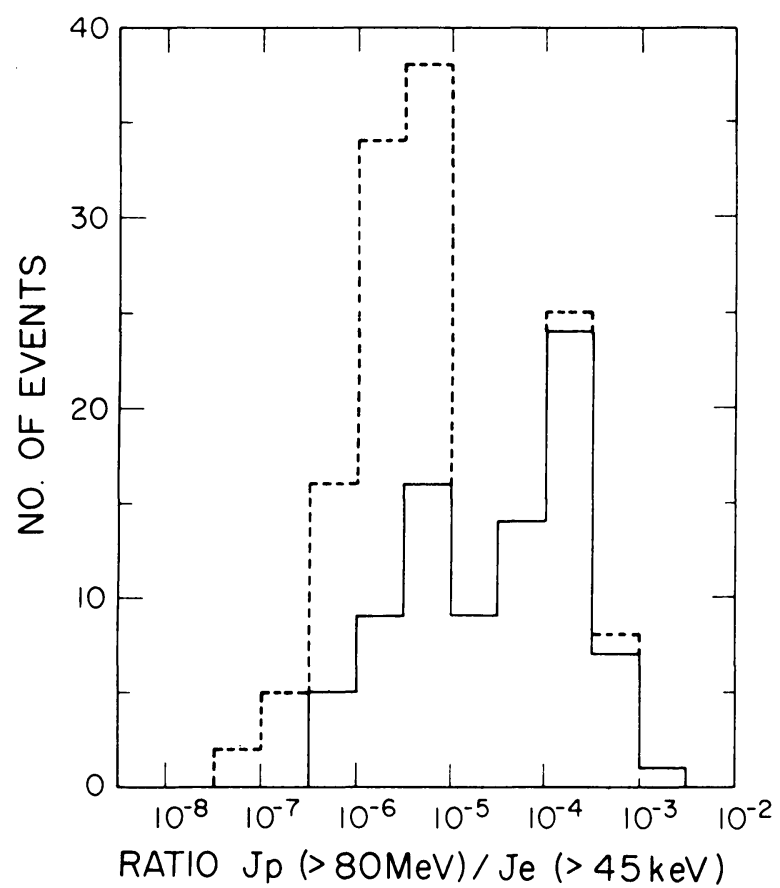

Fig. 11. The number of events plotted vs the ratio of protons to electrons of the same velocity. The solid curve is constructed from all the points for which a proton flux was actually observed. The dotted curve includes those points for which only an upper limit to the proton flux was obtained. Two peaks appear in the distribution, one at ratios of $\sim 10^{-6}$ to $10^{-5}$ and the other at ratios of $10^{-4.5}$ to $10^{-3}$. The peak at ratios of $10^{-4.5}$ to $10^{-3}$ includes almost all energetic proton events as defined by the criterion given in the text.

electron flux vs the maximum $>10 \mathrm{MeV}$ proton flux for all impulsive events which were observed by IMP or OGO spacecraft ionization chambers (S. R. Kane, 1974, private communication). These chambers were used because of their high sensitivity to $\sim 10-15 \mathrm{MeV}$ protons; events with maximum $>10 \mathrm{MeV}$ proton flux of $3 \times 10^{-3}$ $\left(\mathrm{cm}^{2} \mathrm{~s} \mathrm{sr}\right)^{-1}$ could be picked out of the counting rates. No attempt was made to correct for any propagation effects so a great deal of scatter is present. Many events, including a majority of the pure electron events, were unaccompanied by a detectable proton increase. Figure 11 plots the number of events versus the ratio of proton flux above $80 \mathrm{MeV}$ to electron flux, above $45 \mathrm{keV}$ (i.e., above equal particle velocities), 
assuming an $E^{-3}$ spectrum for the protons. Two peaks are evident in the distribution, one between ratios of $10^{-3}$ and $10^{-4.5}$ and the other beyond $10^{-5}$. Almost all the large proton events - events with a second acceleration phase - fall in the $10^{-3}$ to $10^{-4.5}$ peak, while the micro-events fall into the $>10^{-5}$ portion. The events in the second group may be flash phase proton events. However, we note that most electron events (65 out of 95) are unaccompanied by any proton fluxes above the ionization chamber threshold. Confirmation of the micro-event proton origin in the flash phase could be obtained if an analysis of the velocity dispersion shows that the injection time is indeed coincident with the flash phase. The low fluxes may preclude sufficiently accurate analysis. Tentatively, we conclude that although some flash phase events may produce a small flux of energetic protons with velocities comparable to the electron observed from the same event, the efficiency of the acceleration is much lower than in usual proton events.

\section{The Flash Phase Acceleration Mechanism}

For many flares the acceleration of electrons to $\sim 5-100 \mathrm{keV}$ energies must be the dominant form of energy release for the flare. We can place stringent requirements on the electron acceleration mechanism in the flare:

(1) The mechanism must be highly efficient in the sense that most of the flare energy is contained in the accelerated electrons.

(2) The time scale for the acceleration is $\lesssim$ few s, and more likely $\lesssim 1 \mathrm{~s}$.

(3) Acceleration occurs over a period of 10 to $100 \mathrm{~s}$, possibly in the form of a group of short pulses.

(4) The average accelerated electron energy varies through the event, going from low at onset to high at time of maximum acceleration to low again at the end.

(5) The mechanism preferentially accelerates electrons to $\sim 5-100 \mathrm{keV}$ energy while accelerating very few, if any protons to comparable velocities.

(6) Approximately $10^{36}$ electrons are accelerated above $\sim 20 \mathrm{keV}$.

(7) The spectrum of the accelerated electrons is rarely harder than $\mathrm{d} n / \mathrm{d} E \propto E^{-2.5}$.

(8) The acceleration region is located in the lower corona at densities of $n_{\mathrm{i}} \lesssim 10^{10}$ $\mathrm{cm}^{-3}$.

(9) Only 0.1 to $1 \%$ of the accelerated electrons escape to the interplanetary medium.

Of these restrictions, clearly the first one is the most difficult to meet. Basically the flare mechanism must be such as to accelerate large numbers of electrons as the primary energy dissipation process. This high efficiency would rule out stochastic or resonant acceleration processes.

We note here that in most flare mechanisms (see Sweet, 1969, for review) there arise large currents and current densities. Laboratory studies of plasmas carrying substantial currents have shown that very efficient and rapid energization of the plasma electrons through collective wave-particle effects results for large enough values of the current density (Hamberger et al., 1971). 
This situation occurs for current densities $j=n_{\mathrm{e}} v_{\mathrm{d}}$ corresponding to drift velocities $v_{\mathrm{d}}$ greater than the electron thermal speed

$$
v_{\mathrm{d}} \gtrsim v_{\mathrm{th}}=\left(\frac{k T_{\mathrm{e}}}{m}\right)^{1 / 2} \text {. }
$$

Under these conditions almost all of the input energy is transferred to the plasma electrons (see Figure 12) thus producing a population of energetic electrons.

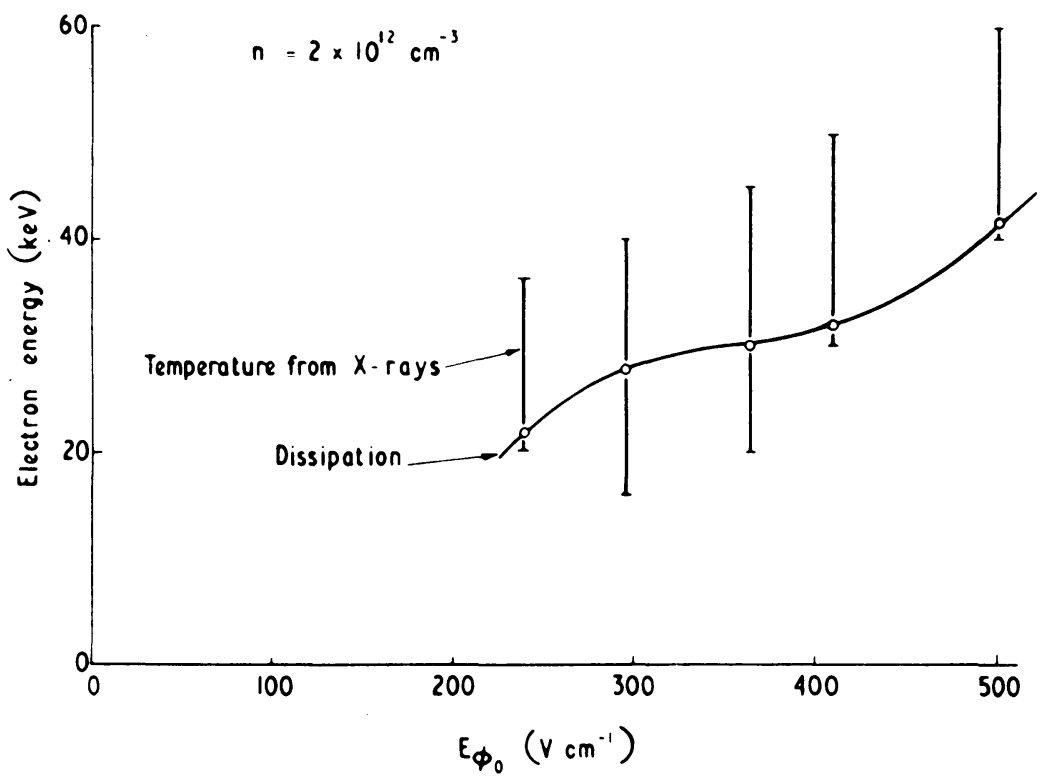

Fig. 12. The results of laboratory experiments in turbulent heating of plasmas, showing that most of the energy fed into the plasma goes to energizing the electrons in the plasma in the Buneman anomalous resistivity region. The smooth curve gives the energy dissipation per plasma electron (obtained from the measured plasma current and applied electric field) while the bars indicate the average electron energy from X-ray measurements. Although this particular measurement is made at a density of $2 \times 10^{12} \mathrm{~cm}^{3}$ the results are valid over a several order of magnitude range of densities (from Hamberger et al., 1971).

In large proton flares the $5-100 \mathrm{keV}$ electrons accelerated in the flash phase are still found to contain the bulk of the total flare energy (Hudson et al., 1975; Hoyng et al., 1975 ), only in these flares the total energy is $\sim 3 \times 10^{32}$ erg above $20 \mathrm{keV}$ and $\gtrsim 10^{33}$ erg for any reasonable choice of the low energy cutoff to the electron spectrum. The rate of energy dissipation must be $\gtrsim 10^{30} \mathrm{erg} \mathrm{s}^{-1}$ and the bulk of the energy must appear as energetic electrons. These conditions are difficult to meet by any magnetic field merging model for flares because the rate of energy dissipation is very rapid, and because most of the energy in magnetic field merging comes out as heating and bulk flow of the plasma rather than as energetic electrons. An attractive alternative to magnetic merging is the following: currents are set up along magnetic field lines by the 
proper motion of the sunspots, and suddenly dissipated through some instability (for example, pinch or magnetic merging) which manages to increase the current density to the critical level for anomalous resistivity to set in. Evidence for this process is provided by the analysis of Tanaka and Nakagawa (1973) of the August 7 flare.

The second phase in large solar flares appears to be the result of a shock wave and ejected material, presumably caused by the explosive heating of the solar atmosphere by the flash phase electrons. When these electrons are few in number, as in small flares, no material is ejected and no second phase ensues.

\section{Acknowledgements}

I wish to acknowledge the support of Professor K. A. Anderson. Discussions with Drs J. C. Brown and S. R. Kane were helpful. This research was supported in part by NASA Grant NGL 05-003-017.

\section{References}

Anderson, K. A. and Lin, R. P.: 1966, Phys. Rev. Letters 16, 1121.

Anderson, K. A. and Winckler, J. R.: 1962, J. Geophys. Res. 67, 4103.

Arnoldy, R. L., Kane, S. R., and Winckler, J. R.: 1968, Astrophys. J. 151, 711.

Berger, M. J. and Seltzer, S. M.: 1964, 'Tables of Energy Losses and Ranges of Electrons and Positrons', NASA SP-3012.

Brown, J. C.: 1971, Solar Phys. 18, 450.

Brown, J. C.: 1972, Solar Phys. 25, 158.

Datlowe, D.: 1975, This volume, p. 191.

Datlowe, D. and Lin, R. P.: 1973, Solar Phys. 32, 459.

Fainberg, J., Evans, L. G., and Stone, R. G.: 1972, Science 178, 743.

Hamberger, S. M., Jancarik, J., Sharp, L. E., Aldcroft, D. A., and Wetherall, A. : 1971, Proc. International Conference Plasma Physics and Controlled Nuclear Fusion, Vol. II, 37.

Holt, S. S. and Ramaty, R.: 1969, Solar Phys. 8, 119.

Hoyng, P., Brown, J. C., Stevens, G., and Van Beek, H. E. : 1975, This volume, p. 233.

Hudson, H. S.: 1972, Solar Phys. 24, 414.

Hudson, H. S., Jones, T. W., and Lin, R. P.: 1975, This volume, p. 425.

Hudson, H. S., Peterson, L. E., and Schwartz, D. A.: 1969, Astrophys. J. 157, 389.

Hundhausen, A. J.: 1972, in C. P. Sonett, P. J. Coleman, Jr., and J. M. Wilcox (eds.), Solar Wind, NASA SP-308, p. 393.

Jackson, J. D.: 1962, Classical Electrodynamics, J. Wiley and Sons, New York.

Kahler, S. W. and Kreplin, R. W.: 1971, Astrophys. J. 168, 531.

Kahler, S. W., Meekins, J. F., Kreplin, R. W., and Bowyer, C. S.: 1970, Astrophys. J. $162,293$.

Kane, S. R.: 1971, Astrophys. J. 170, 587.

Kane, S. R.: 1972, Solar Phys. 27, 174.

Kane, S. R.: 1973a, Bull. A.A.S. 5, 274.

Kane, S. R.: 1973b, in R. Ramaty and R. G. Stone (eds.), High Energy Phenomena on the Sun, NASA SP-342, p. 55.

Kane, S. R.: 1974, Private communication.

Kane, S. R. and Anderson, K. A.: 1970, Astrophys. J. 162, 1003.

Kane, S. R. and Donnelly, R. F.: 1971, Astrophys. J. 164, 151.

Kane, S. R. and Lin, R. P.: 1972, Solar Phys. 23, 457.

Krieger, A., Paolini, F., Vaiana, G. S., and Webb, D.: 1972, Solar Phys. 22, 150.

Lin, R. P.: 1970, Solar Phys. 12, 209.

Lin, R. P.: 1973, in R. Ramaty and R. G. Stone (eds.), High Energy Phenomena on the Sun, NASA SP-342, p. 439.

Lin, R. P. and Hudson, H. S.: 1971, Solar Phys. 17, 412. 
Lin, R. P. and Anderson, K. A.: 1967, Solar Phys. 1, 446.

Lin, R. P., Evans, L. G., and Fainberg, J.: 1973, Astrophys. Letters 14, 191.

McDonald, F. B. and Van Hollenbecke, M.: 1973, in R. Ramaty and R. G. Stone (eds.), High Energy Phenomena on the Sun, NASA SP-342, p. 404.

McKenzie, D. L., Datlowe, D. W., and Peterson, L. E.: 1973, Solar Phys. 28, 175.

Peterson, L. D., Datlowe, D. W., and McKenzie, D. L.: 1973, in R. Ramaty and R. G. Stone (eds.), High Energy Phenomena on the Sun, NASA SP-342, p. 132.

Smith, H. J. and Smith, E. P.: 1963, Solar Flares, MacMillan Co., New York.

Sweet, P. A.: 1969, Ann. Rev. Astron. Astrophys. 7, 149.

Syrovatskii, S. I.: 1969, in C. de Jager and Z. Švestka (eds.), Solar Flares and Space Research, NorthHolland Publ. Co., Amsterdam, p. 346.

Tanaka, K. and Nakagawa, Y.: 1973, Solar Phys. 33, 187.

Takakura, T.: 1972, Solar Phys. 26, 151.

Thomas, R. J. and Neupert, W. M.: 1971, Bull. A.A.S. 3, 264.

Trubnikov, B. A.: 1965, Rev. Plasma Phys. 1, 105.

Van Allen, J. A. and Krimigis, S. M.: 1965, J. Geophys. Res. 70, 5737.

Vorpahl, J. and Zirin, H.: 1970, Solar Phys. 11, 285.

Wild, J. P., Smerd, S. F., and Weiss, A. A.: 1963, Ann. Rev. Astron. Astrophys. 1, 291. 